\title{
Toward elucidating the mechanism of lytic polysaccharide monooxygenases: Chemical insights from X-ray and neutron crystallography \\ Gabriela Schroder ${ }^{1}$, Flora Meilleur ${ }^{2}$ \\ ${ }^{1}$ No affiliation given ${ }^{2}$ No affiliation given \\ gcschrod@ncsu.edu
}

Lytic polysaccharide monooxygenases (LPMOs) are copper-center enzymes involved in the oxidative cleavage of the glycosidic bond. LPMOs are responsible for chain disruption of crystalline cellulose, thereby increasing the accessibility of the carbohydrate substrate to cellulases for hydrolytic depolymerization. The enhanced cellulose conversion of biomass due to addition of LPMOs makes them valuable for the generation of biofuels. The LPMO active site is located on the planar enzyme-cellulose binding surface in which a single copper ion is coordinated in a 'histidine-brace' motif composed of a N-terminal histidine and a second conserved histidine residue in the equatorial plane, with a coordinating tyrosine residue in the axial position. The LPMO reaction is initiated by the addition of a reductant and oxygen to ultimately form an unknown activated copper-oxygen species responsible for polysaccharide substrate hydrogen atom abstraction. Previous work in our group on LPMO9D from Neurospora crassa has provided insight into the binding and activation of oxygen at the LPMO active site as well as the role of the protonation state of a second-shell residue His 157 in oxygen-prebinding (O'Dell et al. 2017). The metallocenter of LPMO makes it highly susceptible to radiation damage, particularly photoreduction and radiolysis due to X-ray beam exposure. Neutron protein crystallography provides a non-destructive technique for structural characterization while also allowing the determination of the positions of light atoms such as hydrogen and deuterium which are central to understanding protein chemistry. Neutron cryo-crystallography permits trapping of catalytic intermediates, thereby providing insight into protonation states and chemical nature of otherwise short-lived species in the reaction mechanism. To this end, we collected a cryo-neutron diffraction dataset on an ascorbate-reduced LPMO9D crystal to characterize the reaction mechanism intermediates (Schröder et al. 2021). A second neutron diffraction dataset was collected at room temperature on a LPMO9D crystal exposed to low $\mathrm{pH}$ conditions to probe protonation states under acidic conditions.

\section{References:}

1. O'Dell, W. B. Agarwal, P. K. \& Meilleur, F. (2017). Angew. Chemie - Int. Ed. 56, 767-770.

2. Schröder, G. C. O'Dell, W. B. Swartz, P. D. \& Meilleur, F. (2021). Acta Crystallogr. Sect. F Struct. Biol. Commun. 77, 128133.
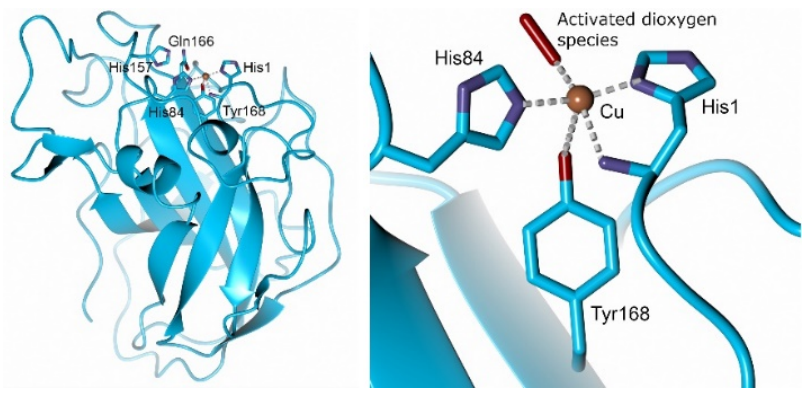

Figure 1 